\title{
Macro H2A.1 immunohistochemical expression in an "in vivo" model of orthodontic dental movement
}

\author{
Claudia Lombardo ${ }^{1}$, Rosalia Leonardi ${ }^{2}$, Sergio Castorina ${ }^{1,3}$, Vera Filetti ${ }^{4}$, Alessandra Ruggeri ${ }^{5}$, Talic Nabeel ${ }^{6}$ and Carla Loreto ${ }^{4 *}$ \\ ${ }^{1}$ Department of Medical, Surgical and Advanced Technological Sciences “G.F. Ingrassia”, University of Catania, Italy \\ ${ }^{2}$ Department of General Surgery and Medical-Surgical Specialities, University of Catania, Italy \\ ${ }^{3}$ Mediterranean foundation "GB” Morgagni Catania, Italy \\ ${ }^{4}$ Department of Biomedical and Biotechnological Sciences, Human Anatomy and Histology, PREDICT, University of Catania, Italy \\ ${ }^{5}$ Department of Biomedical and Neuromotor Science, Human Anatomy, University of Bologna, Italy \\ ${ }^{6}$ Department of Anatomy and Cell Biology, University of Illinois of Chicago, California, USA
}

\begin{abstract}
Aim: The present study aimed to verify the histone MacroH2A.1 immunohistochemical expression and localization after orthodontic force application on rat maxillary molars to deep insight the molecular mechanisms involved in tooth movement.

Materials and Methods: Sixteen Sprague-Dawley rats were used as previously described. Tooth movement was induced, and rats were then killed. Molar-bearing segments of alveolar bone were cut from each side and further fixed in paraformaldehyde, decalcified, and then dehydrated. Specimens embedded in paraffin, including the crestal areas mesial and distal to the maxillary molars, were cut by microtome and processed for immunohistochemistry to detect macroH2A. 1 according to literature protocols.

Results: On the compression side, MacroH2A.1 was strongly immunostained in the spindle round cells of PDL, in cementoblast cells of root teeth and osteoclasts of alveolar bone. Sections also showed vacuolization of odontoblastic layer near to the pulp tissue. Alveolar bone evidenced the presence of marrow spaces. On the tension side, odontoblastic cells near to pulp tissue take spindle shape and showed a Journal of Oral Pathology and Medicine - Proof for Peer Review for Review Only scattered cell immunolabelling. Negative controls showed no immunolabelling.
\end{abstract}

Conclusions: The results of the present study demonstrated overexpression of MacroH2A.1, on the compression side, after orthodontic force appliance suggesting a role of this histone in the remodeling of alveolar bone nonetheless further molecular analysis will be helpful to understand the specific involvement of MacroH $2 \mathrm{~A} .1$ in orthodontic mechanical stress.

\section{Introduction}

Orthodontic displacement is a movement induced by exogenous forces applied to transform a malocclusion into an ideal occlusion. Orthodontic tooth movement (OTM) is a system that combines physiologic alveolar bone modification to mechanical strains with minor reversible injury to the periodontium [1]. Clinicians classify OTM into 3 phases: initial rapid progression caused by pressure force, delay a second phase with no obvious tooth movement, and at last a period of rapid tooth movement [1].

The maxillary bone may seem to be stable and unchanging, but really, it is continually subjected to a cellular turn over managed by the osteoblast and osteoclast cell lines. Alveolar bone remodeling is triggered by a release of calcitonin gene related peptide (CGRP) and substance $P$ in the extracellular fluid that are vasodilators and stimulate plasma extravasation and leukocyte migration [2]. Alveolar remodeling is also a response to mechanical stresses on the bone tissue. From a microscopical point of view, osteoblasts are mononuclear boneforming cells observed at the bone surface while osteoclasts are bone cells with resorbing action that break down bone. Osteoblasts derive from the mesenchymal/mesodermal cells while osteoclasts originate from the hematopoietic/monocyte cells [3-4]. The latter are large multinucleate cells, formed by the fusion of multiple monocytes [5-6].
In the periodontal ligament (PDL) on the surface of lamina dura and in endosteal surfaces of the alveolar bone reside the osteoblasts and osteoclasts that are responsive to mechanical stresses [7]. OTM promotes osteoclasts bone resorption on the PDL compression side while osteoblasts cause bone apposition on the tension side, triggering compression side tooth migration [8-11]. Bone resorption occurs through osteoclastic activity, thus making irregular cavities in bone that later will be filled by newly formed bone owing to osteoblast activity [7]. Moreover, the application of these forces in the course of orthodontic displacement, set off a periodontal inflammatory reaction to PDL that activates in turn, a biological response with synthesis and release of prostaglandins, cytokines, growth factors, neurotransmitters, colonystimulating factors that regulate cellular activities in the compression $v s$. tension areas within the PDL promoting a net outcome of bone resorption at the compression side and bone formation at the tension

${ }^{\star}$ Correspondence to: Carla Loreto, Department of Biomedical and Biotechnological sciences VIA S.SOFIA 87, 95125, CATANIA, Italy; E-mail: carla.loreto@unict.it

Key words: MacroH2A.1, tooth, immunohistochemistry, orthodontic movement

Received: December 02, 2019; Accepted: December 11, 2019; Published: December 16, 2019 
side [1,12-14]. In this context, the PDL plays an essential role in the relationship between the dental root and the surrounding alveolar bone and represents a basal element for the biological changes [15].

Previous studies showed that the PDL is compressed on 3rd day of force application, and from day 7 to 14 , resorption lacunae are detected in the pressure side alveolar bone. Osteoclasts emerged along the alveolar bone edge starting from the 3rd day and then increased in the alveolar resorption craters [16-17].

MacroH2A is a unique histone variant, with a C-terminal nonhistone domain, with a crucial role regarding chromatin domain and gene transcription, and it presents different isoforms [17]. In particular, in vertebrates, there are two MacroH2A isoforms, MacroH2A.1, and MacroH2A.2, which are encoded by different genes [18-19]. MacroH2A.1 is due to the presence of a C-terminal macro domain, and it performs an important role in transcription regulation, DNA replication, DNA repair, chromosomal stability, and stable $\mathrm{X}$ chromosome inactivation. DNA accessibility is established through a series of post-translational histones alterations, called histone code, and nucleosome remodeling [20]. The histone variant macroH2A.1 represents a barrier to the cellular reprogramming process. It obstructs the binding of transcription factors and inhibits with SWI/SNF remodeling activity (Switch/Sucrose Non-Fermentable), inducing a hypoacetylated chromatin state. Also, isoform macroH2A.1 ties ADP-ribose and O-acetyl-ADP-ribose and may be implicated in ADPribose-mediated chromatin modulation [21].

Although several lines of evidence suggest that at least some histone modifications may affect bone remodeling in tumor metastasis, actually, there is no data about histone variants expression during bone remodeling due to the application of orthodontic stress. Even if the molecular mechanisms that regulate tooth movement are studied mainly, MacroH2A contribution has never been considered in the alveolar bone. Thus, the purpose of our work was to verify MacroH2A.1 immunohistochemical expression and localization during an in vivo orthodontic force application to deep insight the molecular mechanisms involved in tooth movement.

\section{Materials and methods}

\section{Study sample}

Sixteen Sprague-Dawley rats (weighing 120-200 g) were examined in the current investigation and according to previous experimental techniques [22]. Tooth movement was caused by putting elastic bands between the maxillary first and second molars [23-24]. This treatment generates compression and tension areas in the alveolar cavity around molar roots and produces a force of about 5 Newton [25].

The elastic bands were inserted for $24 \mathrm{~h}$ using anesthesia based on ketamine $(90 \mathrm{mg} / \mathrm{kg}$ ) (Abbott Laboratories, NorthChicago, IL) and Xylazine.

A fixative solution of $4 \%$ paraformaldehyde, $\mathrm{pH} 7.2$ was used by intracardiac perfusion in order to kill rats. After perfusion, the maxillae were cut free. Molar-bearing segments of alveolar bone were dissected from each side and besides fixed in isolate containers, including $50 \mathrm{ml}$ of $4 \%$ paraformaldehyde overnight at $4^{\circ} \mathrm{C}$. Specimens were decalcified in $10 \%$ ethylenediaminetetraacetic acid (EDTA) (Sigma, St. Louis, $\mathrm{MO}$ ), $\mathrm{pH} 7.2$, for 6-8 weeks and then dehydrated through ethanol rising concentrations. After, the specimens were embedded in paraffin wax as from regular protocol. Maxillary molar sections, 4-6-mm thick, were cut by a microtome sidelong, including mesial and distal crestal tooth areas.

\section{Histochemistry}

Osteoclasts were identified by histochemical staining with Tartrate resistant acid phosphatase (TRAP) which is a well-established marker for the chondro-/osteoclast lineage. Sections were firstly deparaffinized, washed in PBS and incubated, for 30-40 min at room temperature, with a solution containing $50 \mathrm{mM}$ sodium acetate (pH 5.2), $0.15 \%$ NaphtolAS-TR-phosphate, $50 \mathrm{mM}$ sodium tartrate, and $0.1 \%$ Fast Red T.R. (Sigma Aldrich Chemie Gmbh, Taufkirchen, Germany), and these processes have allowed to show TRAP activity. Finally, the sections were washed in PBS and counterstained.

\section{Immunohistochemistry}

Tooth tissue sections were deparaffinized, rehydrated, and treated for MacroH2A.1 immunohistochemistry detection according to literature protocols [24]. Slides were incubated with $0.3 \% \mathrm{H}_{2} \mathrm{O}_{2} /$ methanol in order to inhibit endogenous peroxidase activity and then washed for $20 \mathrm{~min}$ with phosphate-buffered saline (PBS; Sigma, Milan, Italy). Sections were heated ( $5 \mathrm{~min} \times 3)$ in capped polypropylene slideholders with citrate buffer (10 mM citric acid, $0.05 \%$ Tween $20, \mathrm{pH}$ 6.0; Bio-Optica, Milan, Italy), using a microwave oven $(750 \mathrm{~W})$. Then slides were incubated for 1 hour, with the primary antibody with $5 \%$ bovine serum albumin (BSA; Sigma, Milan, Italy) in PBS in a humid chamber. BSA acts as a blocking agent to prevent non-specific binding of the antibody. After, sections were incubated with a rabbit polyclonal macroH2A.1 (Ab-1, clone VIIIA2, Neomarkers, Lab Vision, Fremont, CA, USA), at a 1:20 working dilution, overnight at $4^{\circ} \mathrm{C}$. Immune complexes treated with a biotinylated link antibody were identified with peroxidase-labeled streptavidin, both incubated for $10 \mathrm{~min}$ at room temperature (LSAB+ System-HRP, K0690; Dako, Glostrup, Denmark). The immunostaining was visualized by 2 minutes incubation in $0.1 \%$ 3,3'-diaminobenzidine and $0.02 \%$ hydrogen peroxide solution (DAB substrate Chromogen System; Dako, Denmark). Sections were then counterstained with Mayer's hematoxylin (Histolab Products $A B$, Göteborg, Sweden) mounted in GVA (Zymed Laboratories, San Francisco, CA, USA) and observed and photographed with an Axioplan Zeiss light microscope (Carl Zeiss, Oberkochen, Germany) and a digital camera (AxioCam MRc5, Carl Zeiss, Oberkochen, Germany), respectively. Positive controls consisted of tissue breast carcinoma. Negative controls were labeled as described above except that the incubation with the primary antibody was replaced by incubation in PBS alone.

\section{Evaluation of immunohistochemistry}

MacroH2A.1 immunostaining was identified as either negative or positive. Immunohistochemical positive staining was considered as the presence of brown chromogen visualization on the margin of the hematoxylin-stained cell nucleus, distributed within the cell membrane and/or cytoplasm by light microscopy observation [26]. Seven fields, arbitrarily chosen, were examined for morphometric and densitometric analysis. The percentage of areas showing MacroH2A.1 immunostaining was expressed as \% positive, dark brown pixels of the analyzed fields. An image acquisition software (AxioVision Release 4.8.2 - SP2 Software, Carl Zeiss Microscopy GmbH, Jena, Germany) was used to evaluate the intensity staining level exhibited as densitometric count ( pixel $^{2}$ ) of positive, dark brown pixels of the analyzed fields. Results were expressed as mean \pm standard deviation (SD). The statistical significance of the data was thus achieved. Digital micrographs were performed using the Zeiss Axioplan light microscope (Carl Zeiss, Oberkochen, Germany) with a digital camera (AxioCam MRc5, Carl Zeiss, Oberkochen, Germany). 


\section{Statistical analysis}

Statistical analysis was performed using SPSS software SPSS $^{\circ}$ release 16.0, Chicago, IL, USA). Data were analyzed with the Kolmogorov-Smirnov test. Parameters were regularly distributed. The unpaired t-test was used to compare the two means. P-values of less than 0.05 were reputed significantly relevant. Data are presented as mean \pm SD [27].

\section{Results}

In the present study, we considered the histological, histochemical, and immunohistochemical changes stimulated by orthodontic tooth movement. The latter creates compression and tension areas with the following histological features: fibroblasts and fibrillar collagen were radially oriented, the PDL fibers appear narrow and extremely compacted with fibers disoriented both on the tension and compression sides, polynucleate osteoclast cells were also detected only on the compression side. Herein, a moderate vacuolization of the odontoblastic layer (green arrow) near to the pulp tissue, with MacroH2A.1 cells immunolabelling (black arrow) (Figure 1). The alveolar bone showed the presence of enlarged marrow spaces, on the compression side within scattered immunolabelled cells (black arrows) (Figure 2). The $\%$ of stained areas by MacroH2A.1, and densitometric count ( pixel $^{2}$ ), exhibited by dark brown pixels of the tested fields, in the compression side, were considered and compared to MacroH2A.1 immunoexpression of the tension side (Chart 1). From densitometric count, immunoexpression of MacroH2A.1 on the PDL compression side was strongly demonstrated (red color), as in the alveolar bone and in particular in osteoclastic cells (black arrows) (Figure 3). Moreover, densely and packed narrow PDL round cells were immunodetected on the compression side showing a strong MacroH2A.1 immunoexpression with a high \% of stained areas respect to the tension side (p0.001) (Figure 4). Negative controls showed no immunolabelling.

\section{Discussion}

In this study, we demonstrated that MacroH2A.1 plays an important role in the response of alveolar bone and PDL to the orthodontic force applied for tooth movement. Tissue reaction on the compression side,

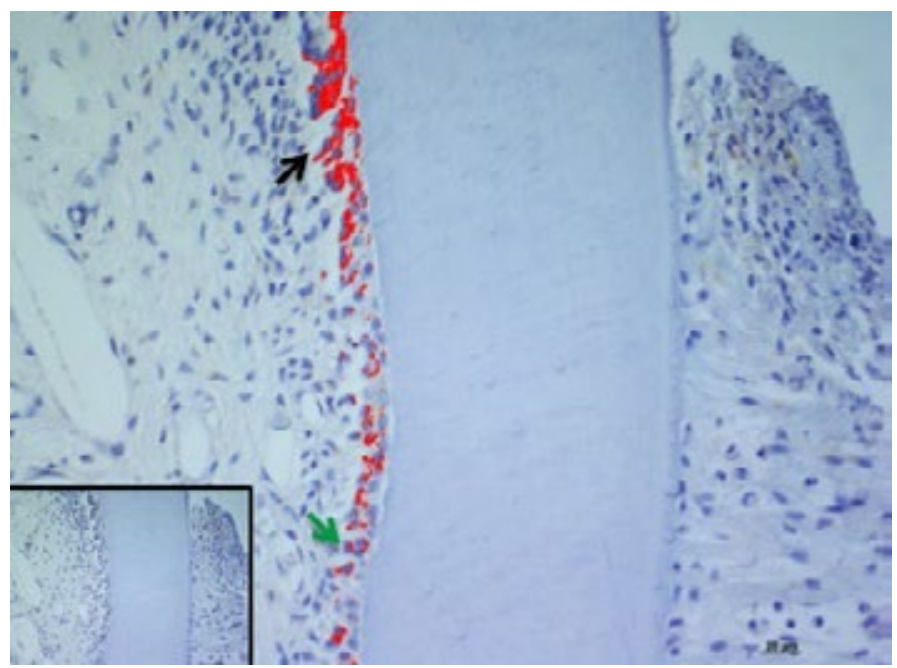

Figure 1. MacroH2A.1 immunoexpression on the compression side of tooth section. Green arrow indicate a moderate vacuolization of odontoblastic layer (OB), black arrow evidence MacroH2A.1 odontoblasts immunolabelling. Amplification: 40X; scale bar: $20 \mu \mathrm{m}$. $113 \times 85 \mathrm{~mm}(300 \times 300$ DPI)

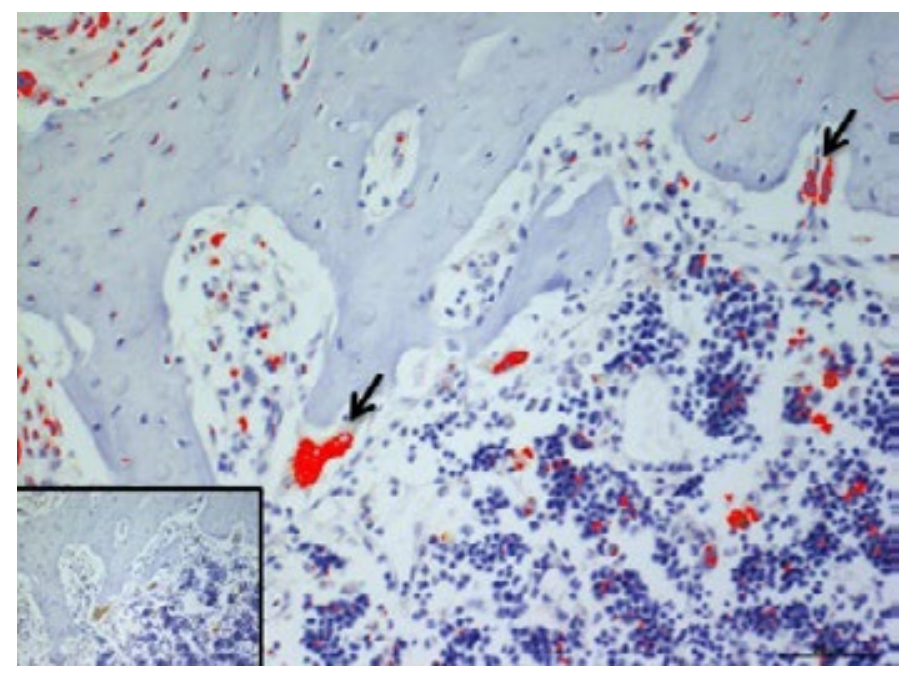

Figure 2. MacroH2A.1immunolocalization on the compression side of the alveolar bone (AB), black arrows evidence scattered immunolabelled cells. Amplification: 40X; scale bar: $20 \mu \mathrm{m} .113 \times 85 \mathrm{~mm}(300 \times 300 \mathrm{DPI})$

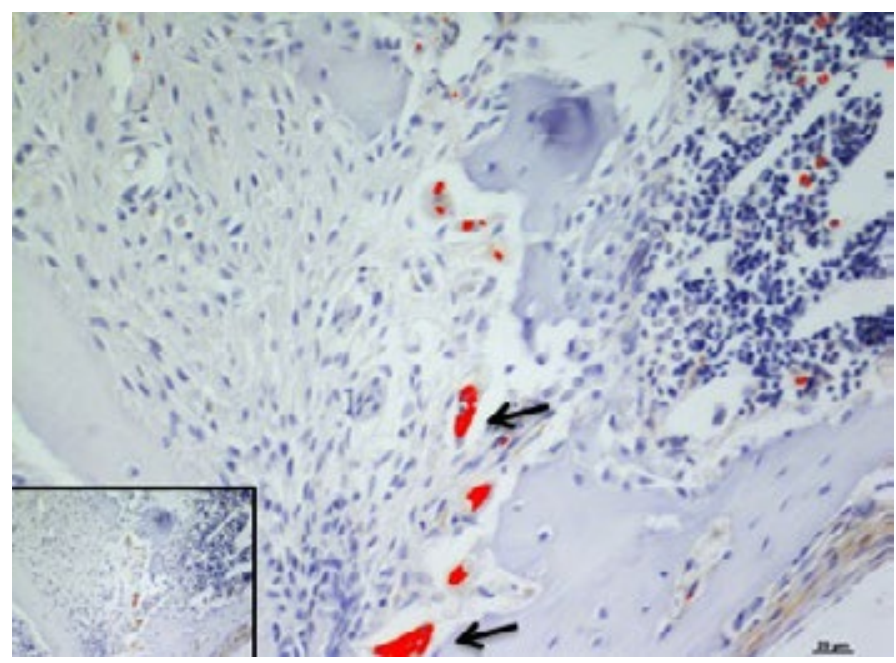

Figure 3. Osteoclastic cells (black arrows) immunoexpression of MacroH2A.1 on the PDL compression side (red color). Amplification: 40X; scale bar: $20 \mu \mathrm{m}$. 113x85mm (300 x $300 \mathrm{DPI})$

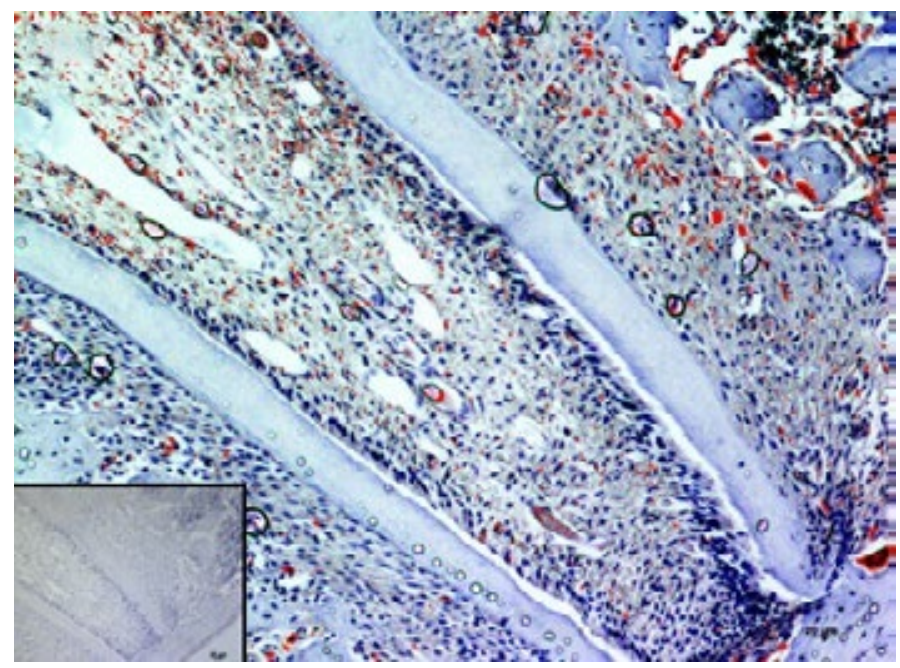

Figure 4. MacroH2A.1 immunodetection on the compression side. Amplification: 20X; scale bar: $20 \mu \mathrm{m} .118 \mathrm{x} 84 \mathrm{~mm}(300 \times 300 \mathrm{DPI})$ 


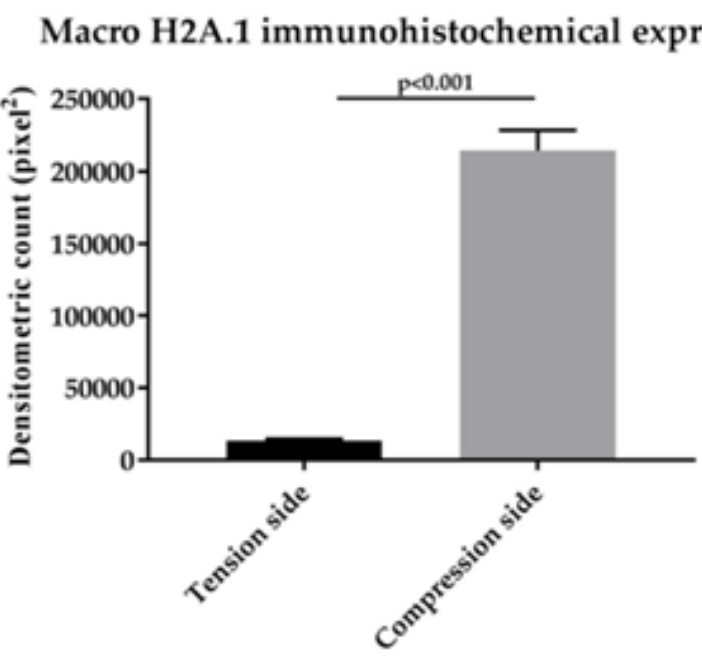

Chart 1. A bar chart representing a comparison of the immunostaining intensity in MacroH2A.1 positive areas of the PDL compression side vs. tension side. Gray bars, high immunostaining; black bars, low immunostaining. Data are presented as mean \pm SD. ${ }^{*} \mathrm{p}$ $<0,001$

during OTM, have been widely studied and as well know over the year, tooth movement is strongly caused by osteoclastic resorption and inflammatory cells that determinate zones of compression and tension, also influencing long-term maintenance of the orthodontic success due to osteogenic differentiation on the tension side [28-31].

Stress from mechanical force is transmitted intracellularly from the extracellular matrix (ECM) via integrins, which induce a signaling cascade through focal adhesion kinases (FAK) to alter gene expression, proliferation, cytoskeletal organization, differentiation, and tissue remodeling [32-34]. Precedent researchers have demonstrated that metalloproteinases (MMPs), ADAMTS, and cathepsin have focal roles in the PDL remodeling due to orthodontic appliance force [29-31,35]. Nevertheless, to date, no studies regarding MacroH2A.1 activity, modulated by application of orthodontic force, have been conducted. Hence, the present study was carried out to deep insight into the molecular mechanisms induced by OTM at an early phase, after 24 $\mathrm{h}$ elastic bands application, and consider the role of MacroH2A.1 at the secreted protein level. Our results pointed out how MacroH2A.1 immunolabeling was high detectable on the compression side, where we demonstrate an increase of MacroH2A.1 immunoexpression within the PDL and in osteoclast cells bone during experimental tooth movement. We demonstrate here that MacroH2A.1 obstacles reprogramming and contributes to maintaining cell identity by catching cells in the initial process stages, namely, at the mesenchymal-to-epithelial transition (MET) in the PDL compression side.

Osteoclasts are multinucleated giant cells that cause and mediate bone resorption [36]. Hematopoietic, myeloid, multipotential precursors differentiate versus mature osteoclasts thank collaboration with macrophage-CSF (M-CSF, also called CSF-1) and RANKL [37]. The present results demonstrated a scattered MacroH2A.1 immunopositivity of hematopoietic cells within the enlarged marrow spaces suggesting a possible immunodetection of osteoclast precursors. The osteoclast development is carried out through a methodical events chain that is not well clarified, as the initial stem cell division and osteoclast progenitor proliferation in hematopoietic tissues, following mononuclear osteoclast precursors migration to bone resorption sites, then differentiation into osteoclast precursors, named preosteoclasts, guided by cell-to-cell interaction with osteoblasts, and finally fusion into multinuclear osteoclasts [38-40]. The percentage and activity of mature osteoclasts regulate the rate at which a tooth can move through the bone [41-42].

Moreover, our results evidenced a moderate MacroH2A.1 immunoreaction of odontoblastic cells of the compression side, probably due to the activation of the reaction dentin (tertiary dentin) during the appliance of force suggesting a role of this histone also in the dentin layer of tooth traction side.

In conclusion, MacroH2A.1 is expressed during the early phases of OTM, especially on the compression side than to the tension side of the PDL. This data could be relevant to understand the biological reactions better and limit the undesirable effects following an orthodontic force, improving the physiology of tooth movement.

\section{Conflict of interests}

The authors report that they have no conflict of interests.

\section{References}

1. Wise GE, King GJ (2008) Mechanisms of tooth eruption and orthodontic tooth movement. J Dent Res 87: 414-434. [Crossref]

2. Hall M, Masella R, Meister M (2001) PDL neuron-associated neurotransmitters in orthodontic tooth movement: identification and proposed mechanism of action. Todays FDA 13: 24-25. [Crossref]

3. Nijweide PJ, Burger EH, Feyen JH (1986) Cells of bone: proliferation, differentiation, and hormonal regulation. Physiol Rev 66: 855-886. [Crossref]

4. Holtrop ME, King GJ (1977) The ultrastructure of the osteoclast and its functional implications. Clin Orthop Relat Res pp: 177-196. [Crossref]

5. Boyle WJ, Simonet WS, Lacey DL (2003) Osteoclast differentiation and activation. Nature 423: 337-342. [Crossref]

6. Teitelbaum SL (2000) Bone resorption by osteoclasts. Science 289: 1504-1508 [Crossref]

7. Jiang N, Guo W, Chen M, Zheng Y, Zhou J, et al. (2016) Periodontal ligament and alveolar bone in health and adaptation tooth movement. Front Oral Biol 18: 1-8. [Crossref]

8. Hakami Z, Kitaura H, Kimura K, Ishida M, Sugisawa H, et al. (2015) Effect of interleukin-4 on orthodontic tooth movement and associated root resorption. Eur $J$ Orthod 37: 87-94. [Crossref]

9. Isola G, Matarese G, Cordasco G, Perillo L, Ramaglia L (2016) Mechanobiology of the tooth movement during the orthodontic treatment: a literature review. Minerva Stomatol 65: 299-327. [Crossref]

10. Dolce C, Malone JS, Wheeler TT (2002) Current concepts in the biology of orthodontic tooth movement. Semin Orthod 8: 6-12.

11. Fill TS, Carey JP, Toogood RW, Major PW (2011) Experimentally determined mechanical properties of, and models for, the periodontal ligament: critical review of current literature. J Dent Biomech 2011: 312980. [Crossref]

12. Zainal Ariffin SH, Yamamoto Z, Zainol Abidin IZ, Megat Abdul Wahab R, Zaina Ariffin Z (2011) Cellular and molecular changes in orthodontic tooth movement Scientific World J 11: 1788-1803. [Crossref]

13. Bartzela T, Türp JC, Motschall E, Maltha JC (2009) Medication effects on the rate of orthodontic tooth movement: a systematic literature review. Am J Orthod Dentofacial Orthop 135: 16-26. [Crossref]

14. Li Y, Jacox LA, Little SH, Ko C-C (2018) Orthodontic tooth movement: The biology and clinical implications. Kaohsiung J Med Sci 34: 207-214. [Crossref]

15. Nishihira M, Yamomoto K, Sato Y (2003) Mechanics of periodontal ligament. In Natali AN, ed. Dental Biomechanics New York: Taylor \& Francis.

16. Zhou J, Yang F, Xu X, Feng G, Chen J, et al. (2018) Dynamic Evaluation of Orthodontically-Induced Tooth Movement, Root Resorption, and Alveolar Bone Remodeling in Rats by in Vivo Micro-Computed Tomography. Med Sci Monit 18: 8306-8314. [Crossref] 
17. Maze I, Noh KM, Soshnev AA, Allis CD (2014) Every amino acid matters: essential contributions of histone variants to mammalian development and disease. Nat Rev Genet 15: 259-271. [Crossref]

18. Gonzales C, Hotokezaka H, Yoshimatsu M, Yozgatian JH, Darendeliler MA, et al. (2008) Force magnitude and duration effects on amount of tooth movement and root resorption in the rat molar. Angle Orthod 78: 502-509. [Crossref]

19. Talbert PB, Henikoff S (2010) Histone variants--ancient wrap artists of the epigenome Nat Rev Mol Cell Biol 11: 264-275. [Crossref]

20. Melters DP, Nye J, Zhao H, Dalal Y (2015) Chromatin Dynamics in Vivo: A Game of Musical Chairs. Genes (Basel) 6: 751-776. [Crossref]

21. Pehrson JR, Fried VA (1992) MacroH2A, a core histone containing a large nonhistone region. Science 257: 1398-1400. [Crossref]

22. Talic N, Evans CA, Daniel JC, George A, Zaki AM (2004) Immunohistochemical localization of alphavbeta3 integrin receptor during experimental tooth movement. $\mathrm{Am}$ J Orthod Dentofacial Orthop 125: 178-184. [Crossref]

23. Waldo CM, Rothblatt JM (1954) Histologic response to tooth movement in the laboratory rat; procedure and preliminary observations. J Dent Res 33: 481-486. [Crossref]

24. Leonardi R, Loreto C, Barbato E, Polimeni A, Caltabiano R, et al. (2007) A histochemical survey of the human temporomandibular joint disc of patients with internal derangement without reduction. J Craniofac Surg 18: 1429-1433. [Crossref]

25. Ren Y, Maltha JC, Kuijpers-Jagtman AM (2004) The rat as a model for orthodontic tooth movement $\bar{a} \epsilon^{\prime \prime}$ a critical review and a proposed solution. Eur J Orthod 26: 483490. [Crossref]

26. Rapisarda V, Caltabiano R, Musumeci G, Castrogiovanni P, Ferrante M, et al. (2017) Analysis of fibulin-3 after exposure to asbestos-like fibers. Environ Res 156: 381-387. [Crossref]

27. Loreto C, Lombardo C, Caltabiano R, Filetti V, Vitale E, et al. (2019) Immunohistochemical expression and localization of MMP-9, MMP-13, E-Cadherin and $\mathrm{Ki}-67$ in road pavers' skin chronically exposed to bitumen products. Histol Histopathol 34: 1141-1150.

28. Leonardi R, Loreto C, Talic N, Caltabiano R, Musumeci G (2012) Immunolocalization of lubricin in the rat periodontal ligament during experimental tooth movement. Acta Histochem 114: 700-704. [Crossref]

29. Leonardi R, Talic NF, Loreto C (2007) MMP-13 (collagenase 3) immunolocalization during initial orthodontic tooth movement in rats. Acta Histochem 109: 215-220. [Crossref]
30. Sorsa T, Ingman T, Mikkonen T (1992) Characterization of interstitial collagenas in gingival crevicular fluid during orthodontic tooth movement in man. In: Davidovitch $\mathrm{Z}$, editor. The biological mechanisms of tooth movement and craniofacial adaptation. Prattville, AL: EBSCO Media.

31. Takahashi I, Nishimura M, Onodera K, Bae JW, Mitani H, et al. (2003) Expression of MMP- 8 and MMP-13 genes in the periodontal ligament during tooth movement in rats. $J$ Dent Res 82: 646-651. [Crossref]

32. Wang HB, Dembo M, Hanks SK, Wang Y (2001) Focal adhesion kinase is involved in mechanosensing during fibroblast migration. Proc Natl Acad Sci U S A 98: 1129511300. [Crossref]

33. Wang JH, Thampatty BP, Lin JS, Im HJ (2007) Mechanoregulation of gene expression in fibroblasts. Gene 391: 1-15. [Crossref]

34. Wang Y, McNamara LM, Schaffler MB, Weinbaum S (2007) A model for the role of integrins in flow induced mechanotransduction in osteocytes. Proc Natl Acad Sci USA 104: 15941-15946. [Crossref]

35. Sternlicht MD, Werb Z (2001) How matrix metalloproteinases regulate cell behavior. Annu Rev Cell Dev Biol 17: 463-516. [Crossref]

36. Lorenzo J, Horowitz M, Choi Y (2008) Osteoimmunology: interactions of the bone and immune system. Endocr Rev 29: 403-440. [Crossref]

37. Jacome-Galarza CE, Lee SK, Lorenzo JA, Aquila HL (2013) Identification, characterization, and isolation of a common progenitor for osteoclasts, macrophages, and dendritic cells from murine bone marrow and periphery. $J$ Bone Miner Res 28 1203-1213. [Crossref]

38. Ash P, Loutit JF, Townsend KM (1980) Osteoclasts derived from haematopoietic stem cells. Nature 283: 669-670. [Crossref]

39. Suda T (1996) Cells of bone: osteoclast generation. In: Bilezikian J, Raisz L, Rodan G, editors. Principles of bone biology. San Diego (Calif): Academic Press.

40. Tran Van PT, Vignery A, Baron R (1982) Cellular kinetics of the bone remodeling sequence in the rat. Anat Rec 202: 445-451. [Crossref]

41. King GJ, Keeling SD (1991) Histomorphometric study of alveolar bone turnover in orthodontic tooth movement. Bone 12: 401-409. [Crossref]

42. Melsen B (1999) Biological reaction of alveolar bone to orthodontic tooth movement Angle Orthod 69: 151-158. [Crossref]

Copyright: (C2019 Lombardo C. This is an open-access article distributed under the terms of the Creative Commons Attribution License, which permits unrestricted use, distribution, and reproduction in any medium, provided the original author and source are credited. 